\title{
Research Paepr \\ Predictors of Social Isolation among Community-dwelling Older Adults in Iran
}

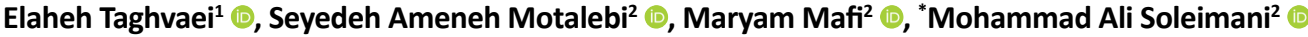

1. Student Research Committee, School of Nursing and Midwifery, Qazvin University of Medical Sciences, Qazvin, Iran

2. Social Determinants of Health Research Center, Research Institute for Prevention of Non-Communicable Diseases, Qazvin University of Medical Sciences, Qazvin, Iran.

\begin{tabular}{l|l}
$\begin{array}{c}\text { Use your device to scan } \\
\text { and read the article online }\end{array}$ \\
Older Adults in Iran (Persian)]. Iranian Journal of Ageing. 2021; 16(2):188-201. https://doi.org/10.32598/sija.16.2.1294.2 \\
dol'https://doi.org/10.32598/sija.16.2.1294.2
\end{tabular}

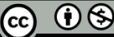

Received: 31 Aug 2020 Accepted: 29 Dec 2020 Available Online: $01 \mathrm{Jul} 2021$

Key words:

Aged, Social isolation, Social support, Disability

\section{A B STRACT}

Objectives Social isolation can lead to depression, reduced life expectancy, and increased death anxiety among older adults. The present study aims to determine the predictors of social isolation in communitydwelling older adults residing in Qazvin, Iran.

Methods \& Materials This descriptive cross sectional study was conducted on 301 older adults in 2019 who were selected using a cluster sampling method. Data were collected using a demographic/clinical checklist, Lubben Social Network Scale, Multidimensional Scale of Perceived Social Support, World Health Organization Disability Assessment Schedule, 5-item World Health Organization Well-Being Index, and Pargament's Religious Coping Questionnaire, which were completed through face to face interview. The collected data were analyzed using multivariable regression analysis.

Results The Mean $\pm S D$ age of participants was $68.63 \pm 7.31$ years. Multivariate regression analysis showed that the significant predictors of social isolation were social support $(\beta=0.333, P<0.001)$, disability $(\beta=$ $0.137, P=0.018)$, and living with spouse $(\beta=0.154, P=0.010)$. The social isolation was reduced by increasing social support, decreasing disability, and living with spouse.

Conclusion Given the role of social support and disability in predicting social isolation in the elderly, it seems that one of the effective factors in reducing social isolation especially in the disable older adults is establishing or strengthening supportive social networks.

\section{Extended Abstract}

\section{Introduction}

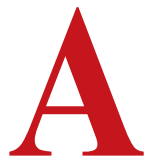

$\mathrm{s}$ the aged population increases, attention to their issues and problems becomes more important [6]. Aging weakens physical and social functioning and increases dependence on others [28]. Older people with physical disabilities are more likely to be lonely and isolated due to lack of social relations and social support $[29,30]$. People who are socially isolated are at higher risk for cardiovascular disease, stroke [14], depression [15] and premature death [17]. Given the growing population of the elderly especially in Iran, addressing the social needs and communication network of the elderly is important. Social isolation in the elderly depends on several individual and social factors; by evaluating and recognizing the factors affecting the social isolation of the elderly, more appropriate decisions and measures can be taken to improve the quality of life of the elderly. This study aims to determine the predictors of social isolation in the elderly living in Iran.

\section{* Corresponding Author:}

Mohammad Ali Soleimani, PhD.

Address: Social Determinants of Health Research Center, Research Institute for Prevention of Non-Communicable Diseases, Qazvin University of Medical Sciences, Qazvin, Iran.

Tel: +98 (912) 2901866

E-mail: soleimany.msn@gmail.com 


\section{Methods}

This descriptive cross-sectional study was conducted on 301 older people in Qazvin, Iran in 2019 who were selected by a cluster sampling method. For sampling, Qazvin was divided into three regions: north, south, and the center. In each regions, 2 parks, 2 mosques, one clinic and one comprehensive urban health center were randomly selected and samples from these settings were selected using a convenience sampling method. In the comprehensive urban health centers, those who had medical records available online were randomly selected. Inclusion criteria were age $\geq 60$ years and ability to communicate verbally.

Data collections tools were a demographic/clinical checklist, Lubben Social Network Scale (LSNS), Multidimensional Scale of Perceived Social Support (MSPSS),
World Health Organization Disability Assessment Schedule (WHODAS), 5-item World Health Organization WellBeing Index (WHO-5), and Pargament's Religious Coping Questionnaire (Brief RCOPE). The LSNS has 12 items rated on a 5-point Likert scale from 0 to 5. Higher scores indicate less social isolation, while scores $<20$ indicate a serious risk of social isolation $[30,31]$. After obtaining ethical approval (Code: IR.QUMS.REC.1398.091), and stating the objectives of the study to the participants and becoming assured of the confidentiality of personal information, they signed an informed consent form. Then, questionnaires were completed via interview. Data were analyzed using multivariate linear regression analysis.

Table 1. Multivariate linear regression model coefficients for social isolation

\begin{tabular}{|c|c|c|c|c|c|}
\hline \multicolumn{6}{|c|}{ Standardized } \\
\hline \multicolumn{2}{|c|}{ Variable } & & $\mathbf{t}$ & $\mathbf{P}$ & Vif \\
\hline \multirow{3}{*}{ Marital status } & Married* & - & - & - & - \\
\hline & & & & & \\
\hline & Single & -0.059 & -0.467 & 0.641 & 0.03 \\
\hline \multirow{3}{*}{ Income level } & Moderate* & - & - & - & - \\
\hline & poor & 0.01 & 0.181 & 0.857 & 1.17 \\
\hline & High & 0.056 & 0.983 & 0.326 & 1.25 \\
\hline \multirow{5}{*}{ Living arrangment } & With spouse* & - & - & - & - \\
\hline & Alone & -0.074 & 0.666 & 0.506 & 2.64 \\
\hline & With spouse/chidlren & 0.154 & 2.609 & 0.010 & 1.33 \\
\hline & With children & 0.121 & 1.149 & 0.252 & 2.18 \\
\hline & With cregiver & 0.041 & 0.694 & 0.488 & 1.32 \\
\hline \multirow{3}{*}{ Smoking } & No* & - & - & - & - \\
\hline & & & & & \\
\hline & Yes & -0.070 & -1.219 & 0.224 & 1.26 \\
\hline \multicolumn{2}{|c|}{ Social support } & 0.333 & 6.095 & $>0.001$ & 1.13 \\
\hline \multicolumn{2}{|c|}{ Disability } & -0.137 & -2.376 & 0.018 & 1.25 \\
\hline \multicolumn{2}{|c|}{ Religious coping } & 0.071 & 1.307 & 0.192 & 1.11 \\
\hline \multicolumn{2}{|c|}{ Psychological well-Being } & 0.084 & 1.429 & 0.154 & 1.32 \\
\hline
\end{tabular}

* Reference group. 


\section{Results}

Out of 301 participants, $74.4 \%(n=244)$ were male and $25.6 \%(n=77)$ were female. Their mean age was $68.63 \pm 7.31$ years. Most of participants were married $(63.8 \%, \mathrm{n}=192)$ with lower than high school education $(40.2 \%, \mathrm{n}=121)$ and a history of chronic disease $(52.5 \%, \mathrm{n}=158)$. Their mean score of LSNS was $27.82 \pm 9.85$ and $15.9 \%(n=48)$ were at serious risk for social isolation. Moreover, their mean scores of WHODAS, RCOPE, WHO-5, and MSPSS was 20.33 \pm 11.16 , $19.30 \pm 4.71,10.06 \pm 5.41$ and $46.70 \pm 9.69$, respectively.

According to the results in Table 1, the variables of social support $(\mathrm{P}<0.001)$, disability $(\mathrm{P}=0.018)$ and living with spouse and children $(\mathrm{P}=0.010)$ had a significant effect on the social isolation of the elderly such that with the increase in social support $(\beta=0.333)$, decrease in disability $(\beta=$ $-0.137)$, and living with spouse and children $(\beta=0.154)$, the scores of social isolation were increased, indicating its improvement.

\section{Discussion and Conclusion}

According to social network theory, social capital creates social relationships with the community and others, which increases social support and protects the person from mental illness as a shield [54]. According to Durkheim's theory, the social relations of the individual increase the social support of the individual, and the increase of social support increases the mental health of the individual; as a result, it increases the sense of pleasure and happiness in life [55]. Therefore, community-based programs in day care centers, focusing on the family and their expansion, communication skills training, providing a suitable environment for receiving social support, and considering effective strategies to eliminate or reduce the impact of physical disabilities on daily life in the elderly can help them to prevent social isolation and rejection and thus increase their quality of life.

\section{Ethical Considerations}

Compliance with ethical guidelines

The study was approved by the Ethics Committee of Qazvin University of Medical Sciences, Qazvin ( Code: IR.QUMS.REC.1398.091). Before to filling out the questionnaires, older participants were assured of the confidentiality of the information obtained and written consent was obtained from all subjects.
Funding

This research did not receive any grant from funding agencies in the public, commercial, or non-profit sectors.

\section{Authors' contributions}

Conceptualization, methodology, research, review of drafts, editing and finalization of writing, review of references: Elahe Taghvaei, Mohammad Ali Soleimani and Seyedeh Ameneh Motalebi; Analysis: Maryam Mafi; Financing: Elahe Taghvaei; Project Management: Mohammad Ali Soleimani.

\section{Conflicts of interest}

The authors declared no conflict of interest.

\section{Acknowledgements}

The authors would like to thank the Vice-Chancellor for Research of Qazvin University of Medical Sciences and all the elderly participated in the study for their support and cooperation. 


\title{
مقاله يخوهشى \\ عوامل ييشبينى كثنده انزواى اجتماعى در سالمندان ساكن جامعه

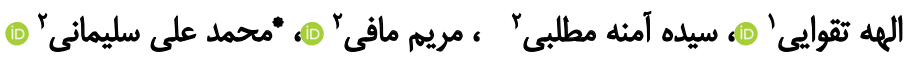 \\ ا. ا.كميته تحقيقات دانشجويى، دانشكاه علوم يزشكى قزوين، قزوين، ايران.

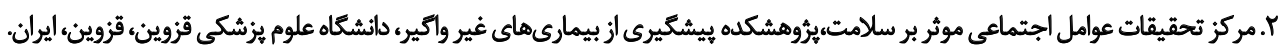

\begin{abstract}
حكSبن

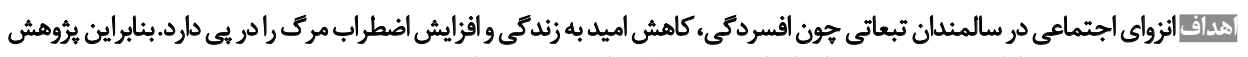

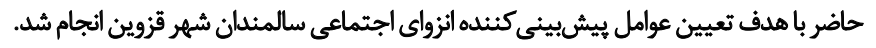

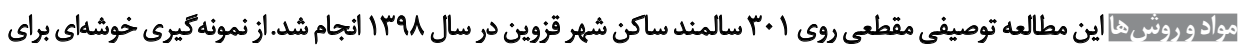

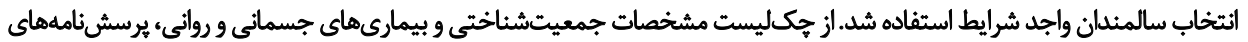

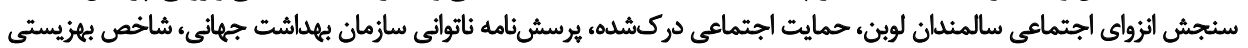

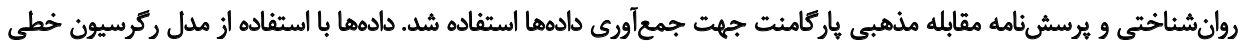

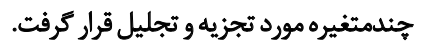

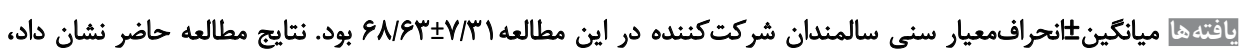

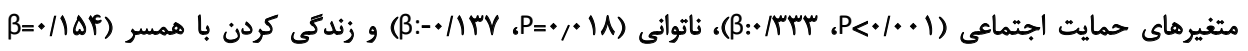

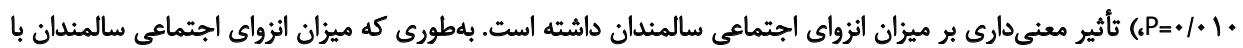

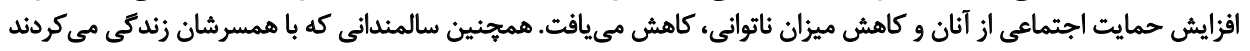

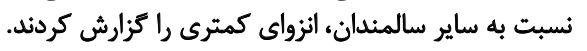

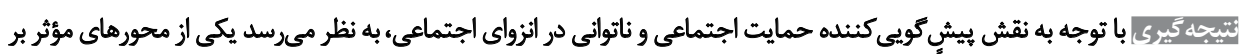

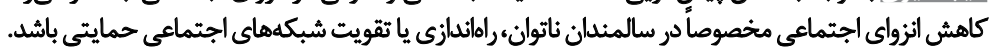

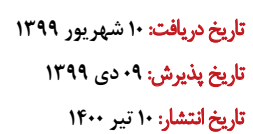

\section{كليدواروها:

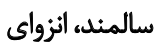

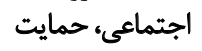

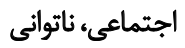

اين قاعده مستثنا نيست. شاخصهاى آمارى نشان ميدهند

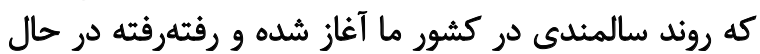

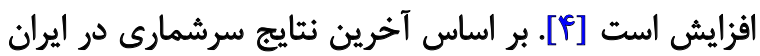

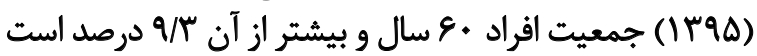

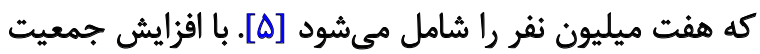

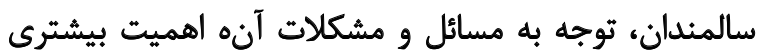

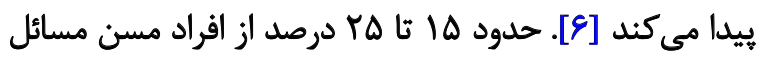

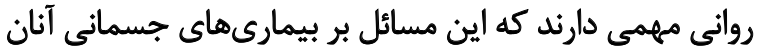

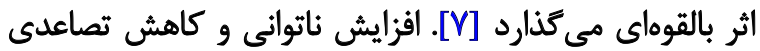

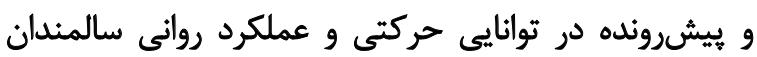

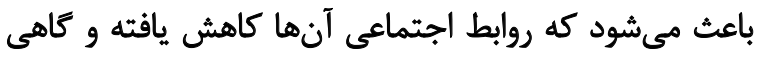

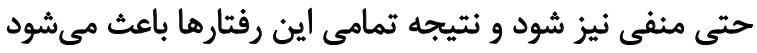

مقدمه

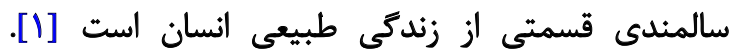

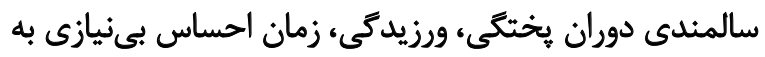

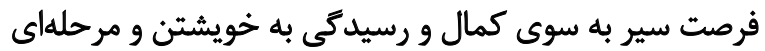

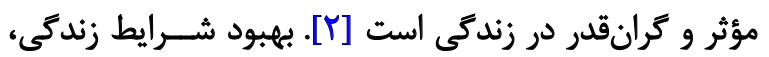

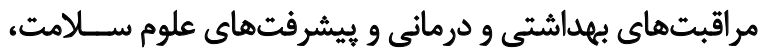

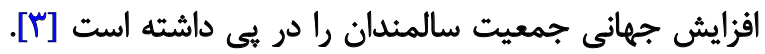

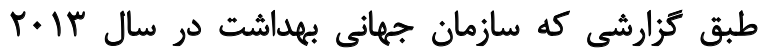

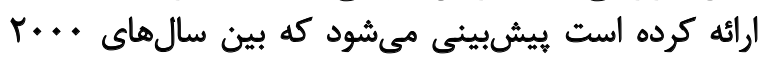

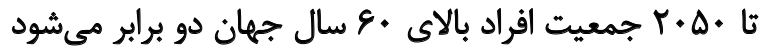

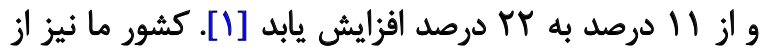

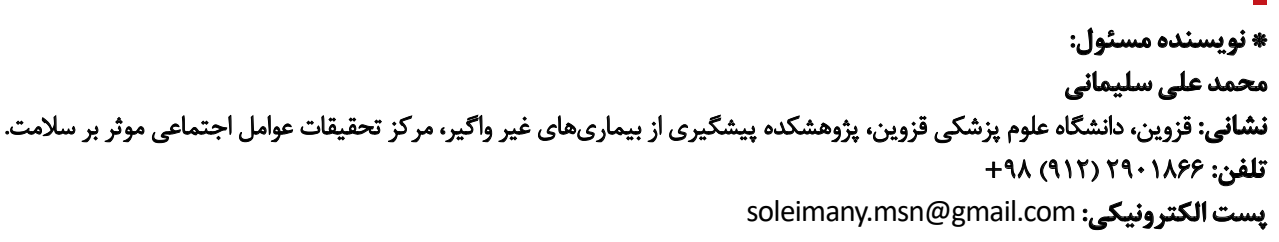


زندگى و تبديل شدن به يك مراقب است [Tr]. در سطح

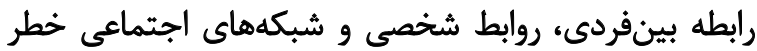

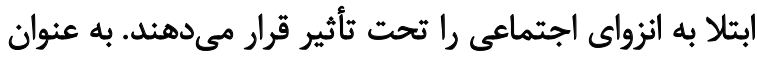

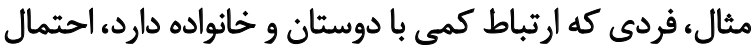

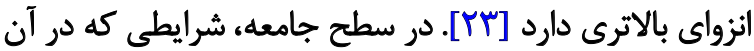

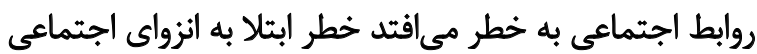

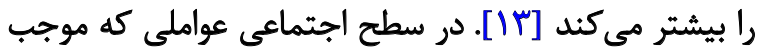

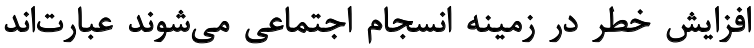

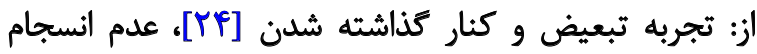

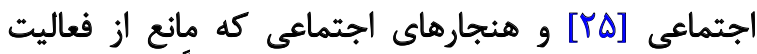

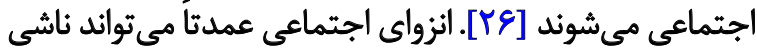

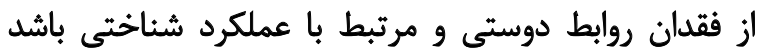

فرايند بيرى سبب تضعيف عملكرد جسمانى و اجتماعى

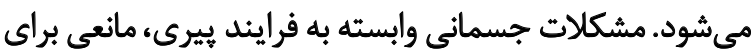

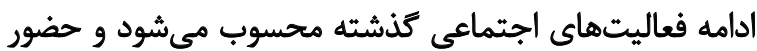

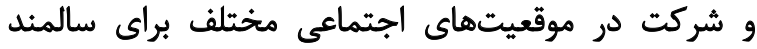

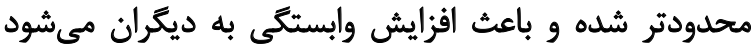

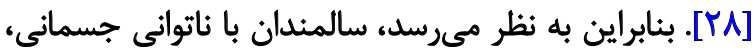

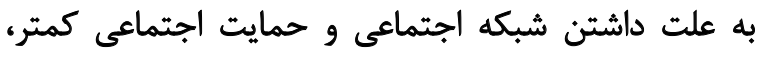

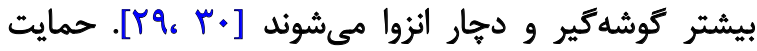

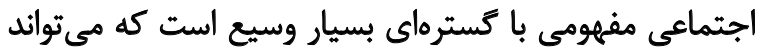

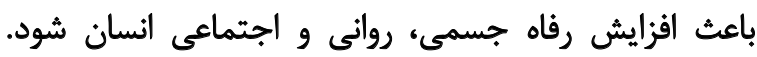

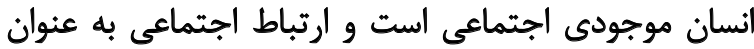

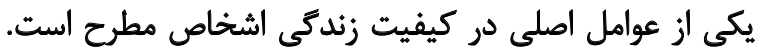

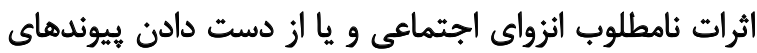

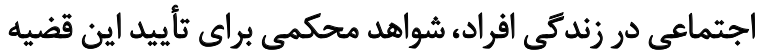

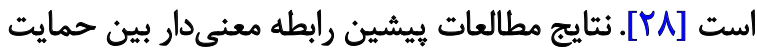

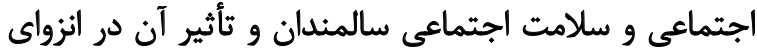

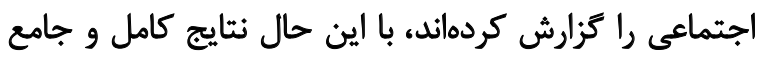

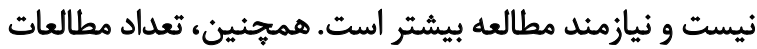

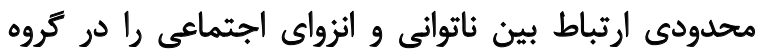
سالمندان مورد بروسي قرار بـ دادهاند.

باتوجه به روند رو به رشد جمعيت سالمندان و اينكه كشورمان

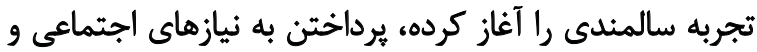

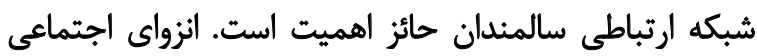

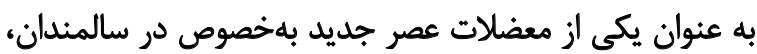

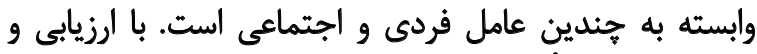

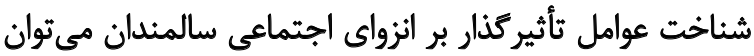

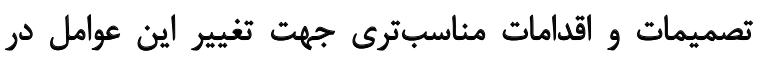

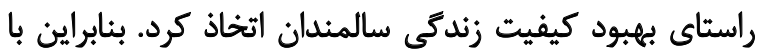

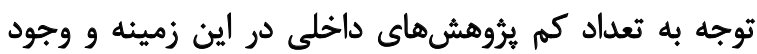

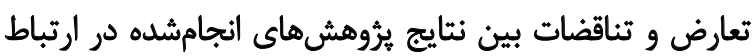

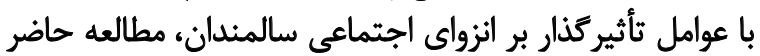

سالمند از تماس و ارتباطات با ديكران خوددارى كند و به انزوا

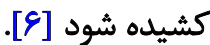

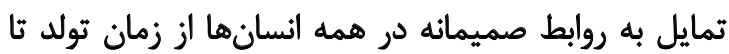

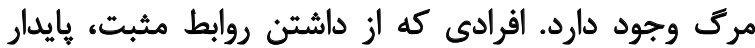

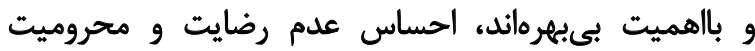

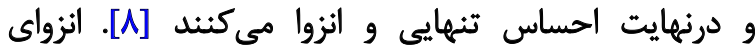

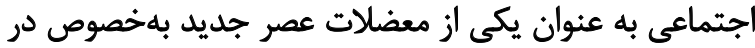

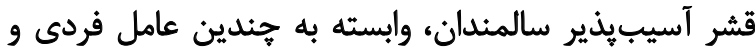

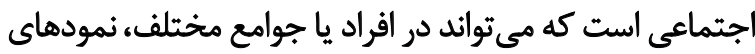

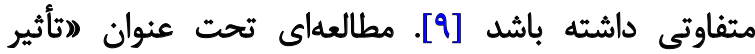

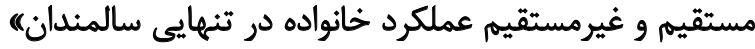

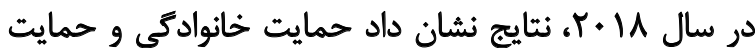

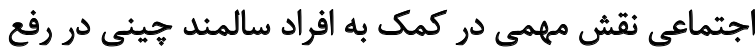

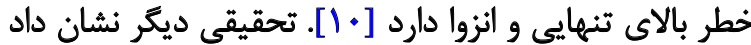

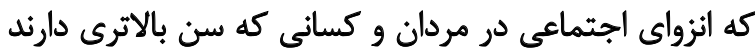

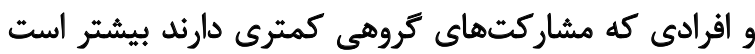

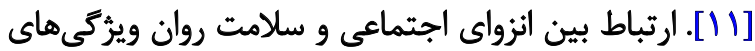

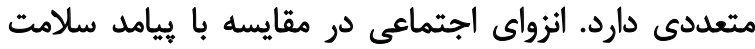

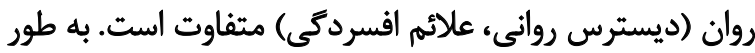

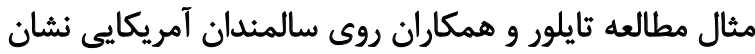

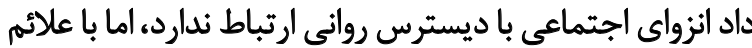

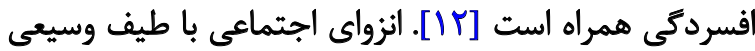

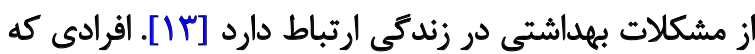

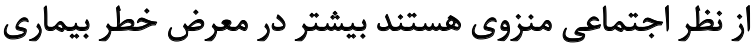

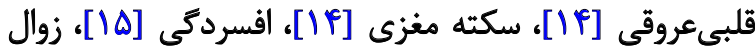

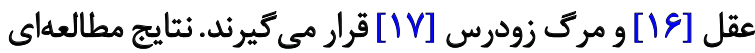

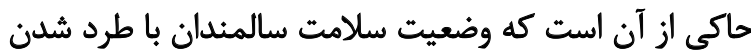

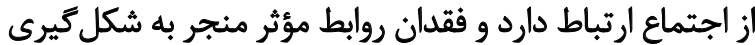

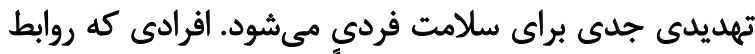

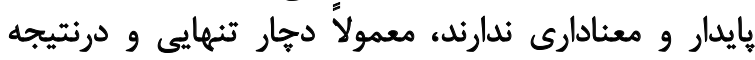

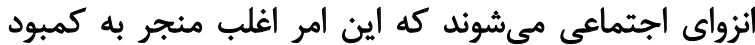

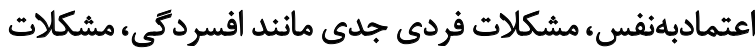

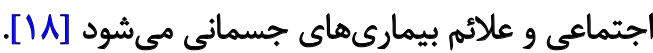
كام آغازين براى حركث در مسيرى كه در آن آن سالمندان

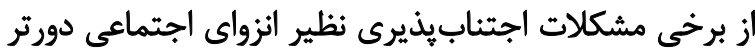

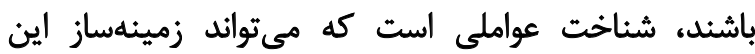

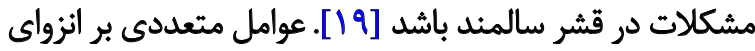

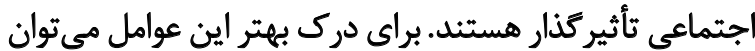

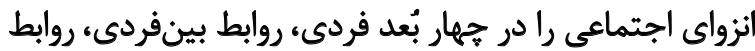

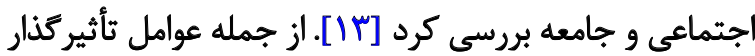

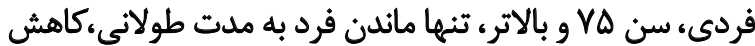

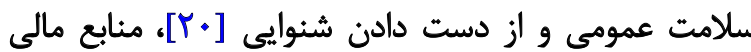

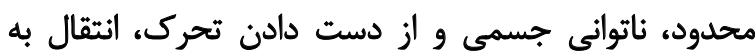

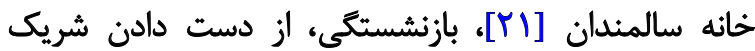




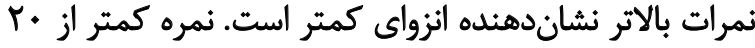

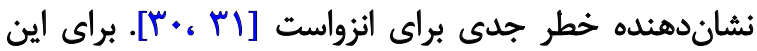

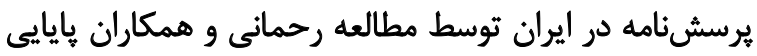

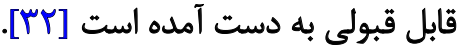

يرسشنامه حمايت اجتماعى دركشده (MSPSS' إين

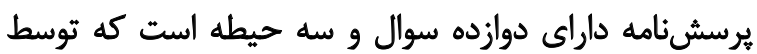

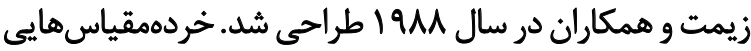

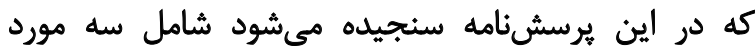

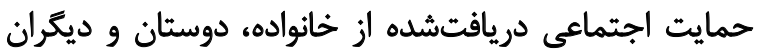

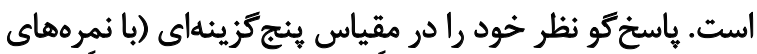

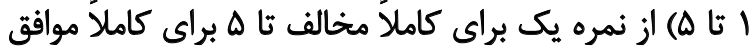

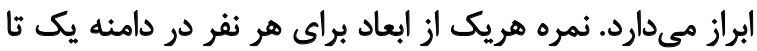

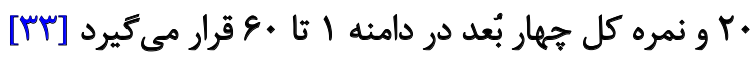

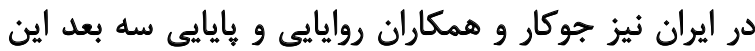

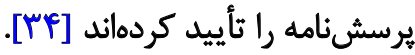

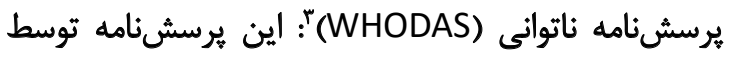

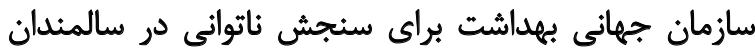

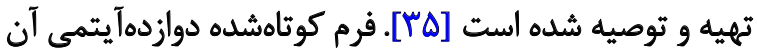

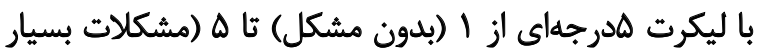

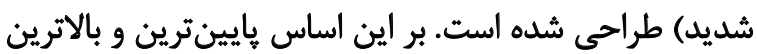

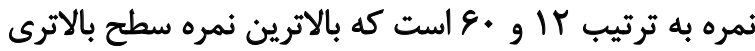

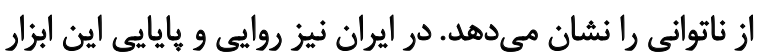

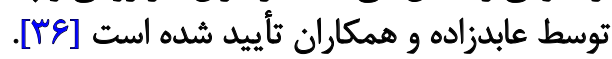

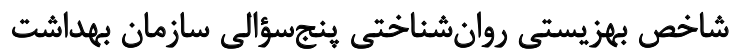

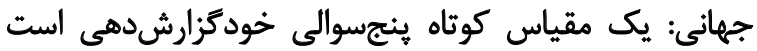

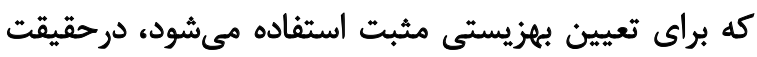

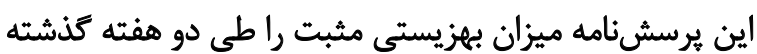

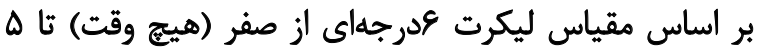

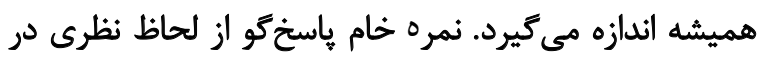

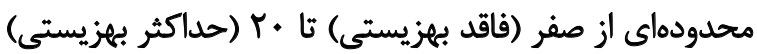

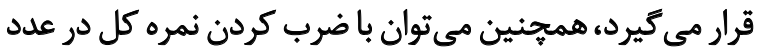

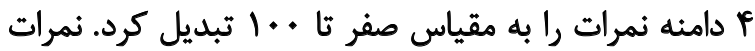

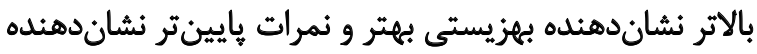

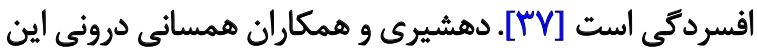

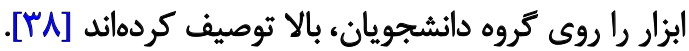

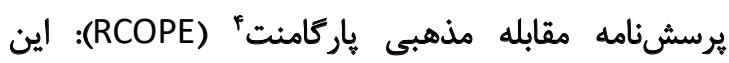

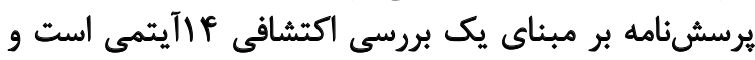

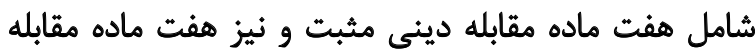

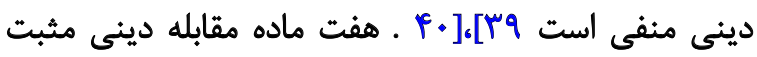

2. Multidimensional Scale of Perceived Social Support

3. WHO Disability Assessment Schedule

4. Pargament
با هدف تعيين ميزان انزواى اجتماعى در سالمندان و شناسايى

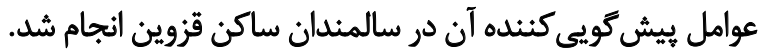

ووش مطأ مالعه

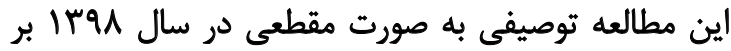

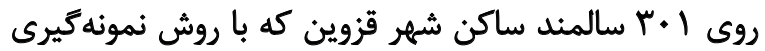

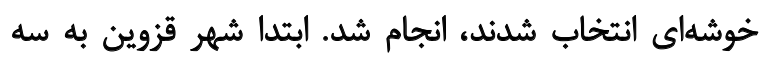

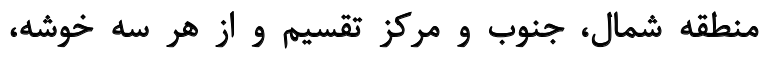

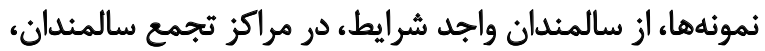

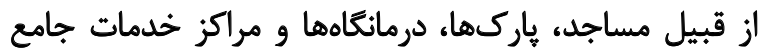

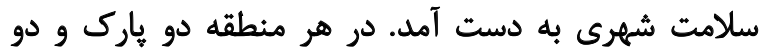

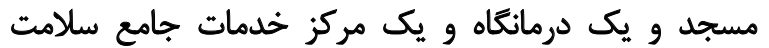

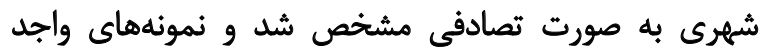

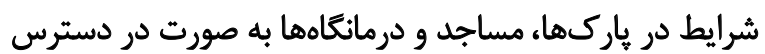

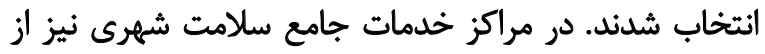

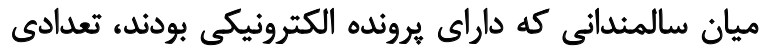

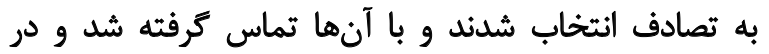

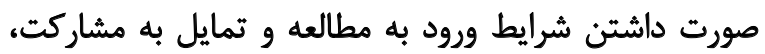

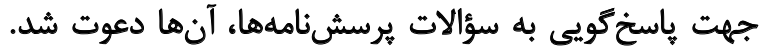

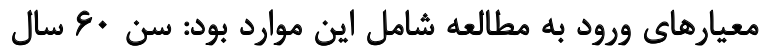

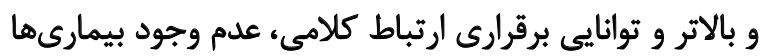

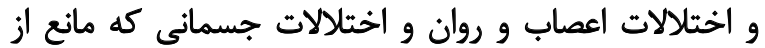

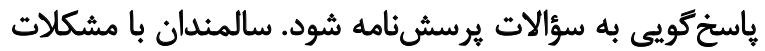

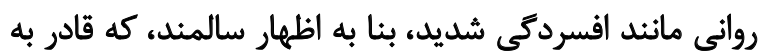
برقرارى ارتباط نبودند از مطالعه كنار كذاشته شدند بند

با توجه به اينكه طبق مطالعه جوى ثاماكا ضريب همبستئى

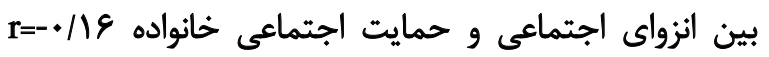

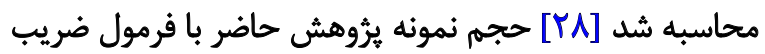

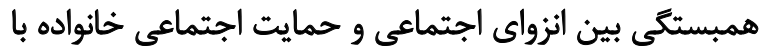

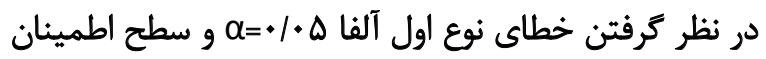

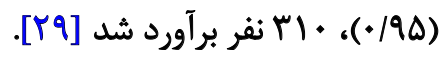

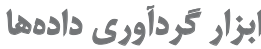

جهت ارزيابى اين موارد، در اين يُروهش از يرسشنامههاى

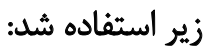

برسشنامه اطلاعات جمعيتشناختى (شامل اطلاعاتى در

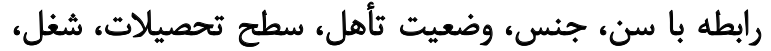

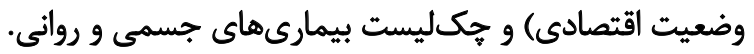

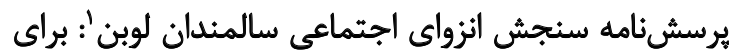

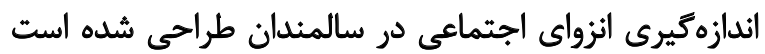
و شامل دوازده سؤال با ليكرت هدرجي درائ انداز صفر ثا ها هاست.

1. Lubben Social Network Scale (LSNS) 
افزايش ناتوانى نمرات انزواى اجتماعى كاهش يافته كه دليلى بر بدتر شدن اوضاع سالمندان است.

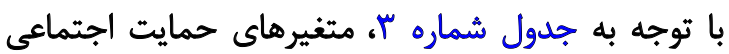

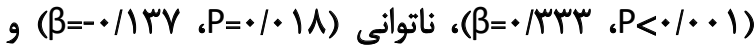

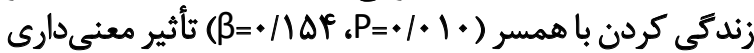

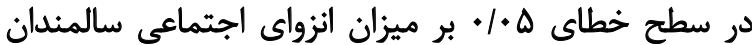

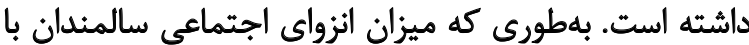

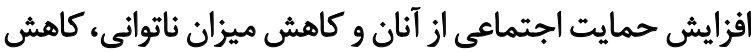

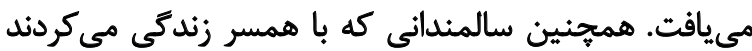

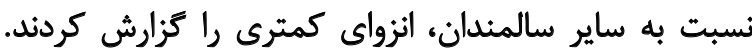

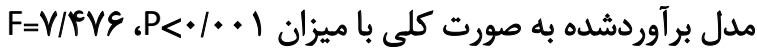

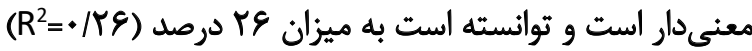

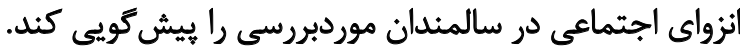

ث)

هدف از مطالعه حاضر، تعيين ميزان انزواى اجتماعى

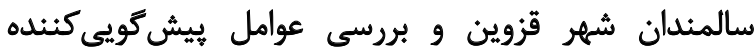

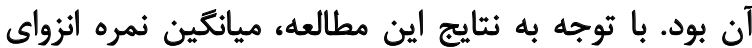

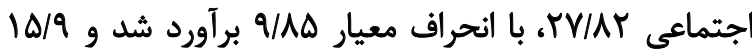

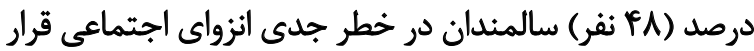

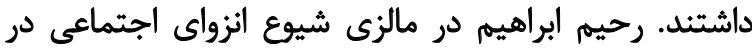

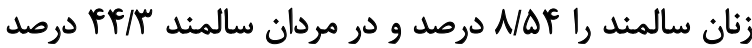

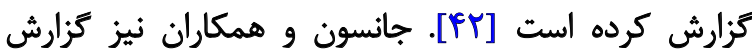

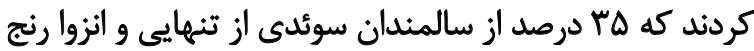

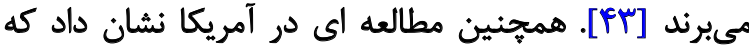

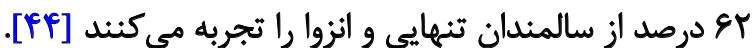

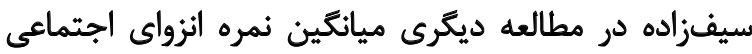

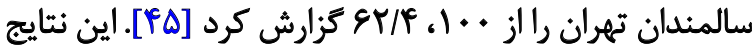

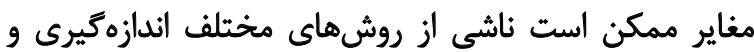

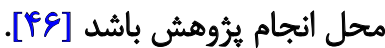

نتايج مطالعه حاضر نشان داد كه با افزايش ميزان حمايت

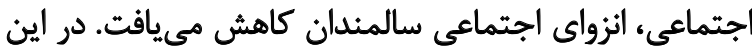

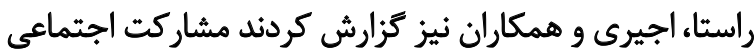

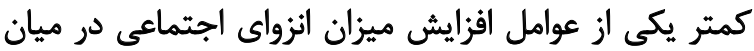

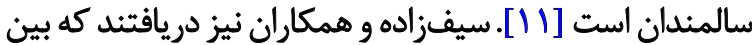

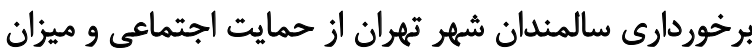

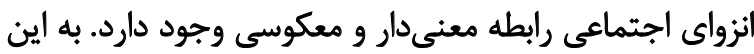

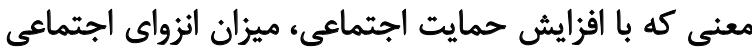

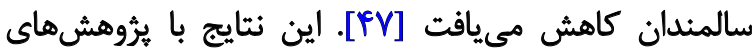

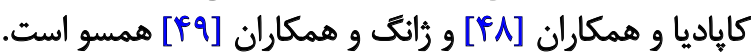

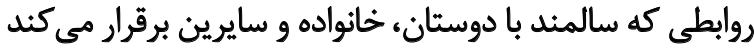

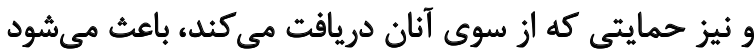

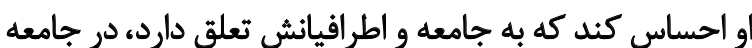
داراى شأن و ارزش است و مورد احترام ديكران است كه اين اين داردان
شامل ييوند معنوى، جستوجوى حمايت معنوى، آموزش دطلبي

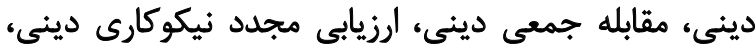

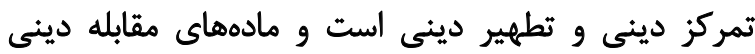

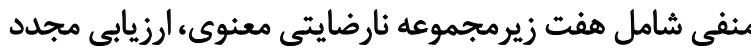

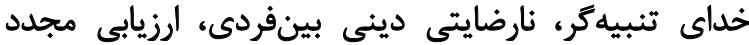

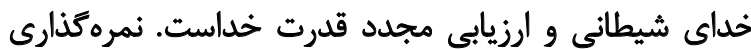

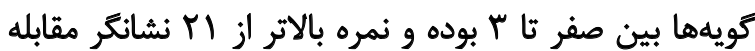

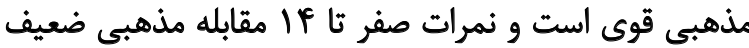

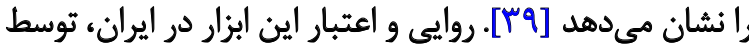

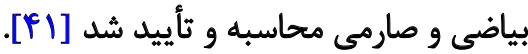

تجزيه و تحليل دادهها

تجزيه و تحليل دادهها با استفاده از نرمافزار SPSS نسخه

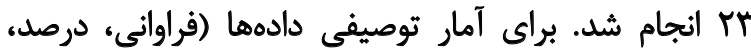

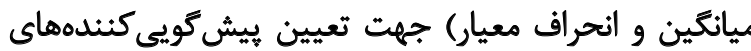

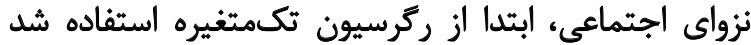

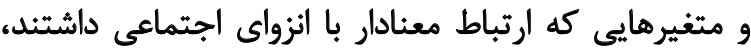

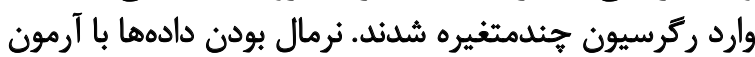

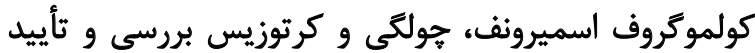

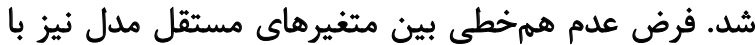

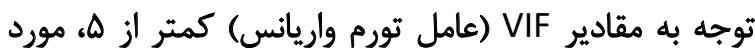

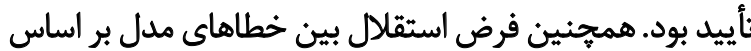

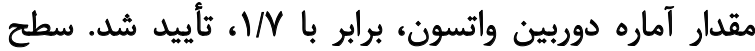

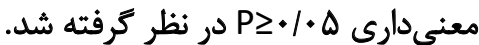

ياقثته ها

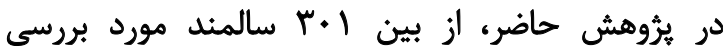

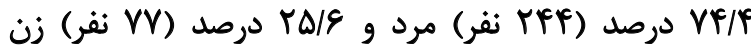

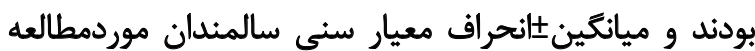

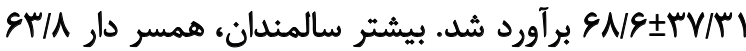

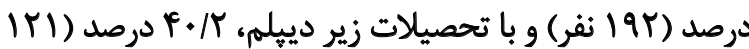
نفر) بودند (جدول شماره ()).

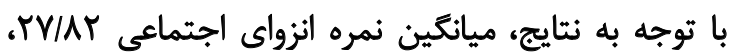

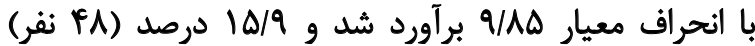
سالمندان در خطر جدى براى انزواى اجتماعى قرار داشتئد

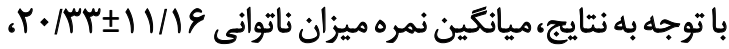

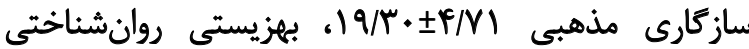
|

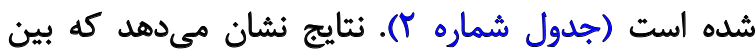
متغير انزواى اجتماعى و متغيرهاى حمائ حمايت اجتماعى و وناتواني

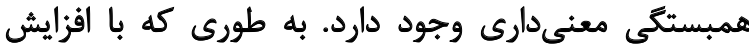

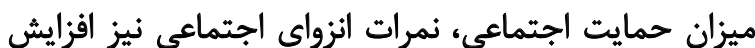

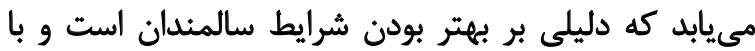


جدول ا. توزيع متغير هاى جمعيتشئاختى در سالمندان موردمطالعه

\begin{tabular}{|c|c|c|}
\hline فُراواني (درصد) & سطح & مثغير \\
\hline$W(T \Delta / P)$ & زن - - - - & \multirow{2}{*}{ جنس } \\
\hline$m \in(M T / P)$ & مرد & \\
\hline $1.9(\mathrm{me/T})$ & بلون همسر & \multirow{2}{*}{ وضعيت تأهل } \\
\hline $197(9 \mathrm{~T} / \mathrm{A})$ & با همسر & \\
\hline D) $(19 / 9)$ & جيى ليسواد & \multirow{3}{*}{ تحصيلات } \\
\hline $\operatorname{lrq}(F T / q)$ & زير دييلم & \\
\hline$M(T \cdot / M)$ & دييلمه و بالاتر & \\
\hline$\Delta)(18 / 9)$ & ضعيف & \multirow{3}{*}{ وضعيث اقتصادى } \\
\hline $\mid E T(\Delta H / \lambda)$ & متوسط & \\
\hline$M(T q / T)$ & خوب & \\
\hline$M F(A)$ & 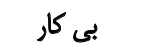 & \multirow{4}{*}{ ت شغل } \\
\hline $1 \% \Delta(\Delta f / \Lambda)$ & بازنشسته & \\
\hline$\Delta A(19 / 4)$ & خانهدار & \\
\hline$\Delta F(I V / q)$ & شاغل & \\
\hline $\operatorname{IPT}(F V / \Delta)$ & دارد & \multirow{2}{*}{ سابقه بيمارى مزمن } \\
\hline $\operatorname{IQN}(\Delta Y / \Delta)$ & تيدارد & \\
\hline $1 f \cdot(18)$ & $1-r$ & \multirow{3}{*}{ تعداد فرزند } \\
\hline $\operatorname{lff}(f \wedge)$ & $f-\gamma$ & \\
\hline $\operatorname{IV}(\varphi)$ & $A-1$. & \\
\hline ميانكين \انحراف معيار & \multicolumn{2}{|c|}{ متغير } \\
\hline ENEM $\pm V / M I$ & \multicolumn{2}{|c|}{ سن } \\
\hline$r g / Q Y \pm T / R Y$ & \multicolumn{2}{|c|}{ شاخص توده بلنى } \\
\hline
\end{tabular}

L

جدول r. ميانكين نمرات متغيرهاى موردبررسى در مطالعه

\begin{tabular}{|c|c|c|c|}
\hline حداكثر & حداقل & ميانكين||نحراف معيار & مثغير \\
\hline q. & $r$ & TV/AYIV/Ad & انزواى اجتماعى \\
\hline$\Delta f$ & ir & $r+/ \pi T \pm 11 / 18$ & ميزان ناتوانى \\
\hline re & - & $19 / \% \cdot \pm t / M$ & سازكارى هذهبي \\
\hline r. & • & $1.1 .8 \pm \Delta / 41$ & بهزيستى روانشناختي \\
\hline \&. & $\pi$ & $P \& / V \cdot \pm q / 9 a$ & حمايت اجتماعي (كل) \\
\hline r. & p & $\mid 8 / r \cdot \pm r / 81$ & حمايت خانواده \\
\hline r. & $p$ & $1 F / \Delta r \pm f / 1 P$ & حمايت دوسثان \\
\hline r. & $p$ & $1 \varepsilon / \cdot V \pm r / \Delta r$ & حمايت ديكران \\
\hline
\end{tabular}


جدول ب. بررسى همزمان يُيش كويى كنندهاى انزواى اجتماعى سالمندان با استفاده از مدل ركرسيون جندمتغيره خطى

\begin{tabular}{|c|c|c|c|c|c|}
\hline Vif & $\mathbf{P}$ & آماره آزمون (t) & ضريب رئرسيون استاندارد (B) & & متغير \\
\hline- & - & - & - & • همسردار & ثأهرل \\
\hline $\mathrm{TlOH}$ & - $|e p|$ &.$- /$ her & $-.1 \cdot \Delta q$ & بلون همسر & \\
\hline- & - & - & - & 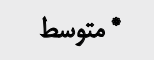 & \\
\hline WIV & - MAY & $\cdot|M|$ & .1 .1 & ضعيف & سطح درأمد \\
\hline I/TO & . MTE & ./ur & $.1 . \Delta 8$ & خوب / غالى & \\
\hline- & - & - & - & 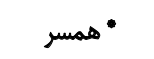 & \\
\hline rlep & $\cdot 10 \cdot 8$ & .1898 & $-+1 * v^{e}$ & تنه & \\
\hline$V / M T$ & $\%$ & $r / 8.9$ & . NAP & همسر / فرزنلد & نحوه ز زندكى \\
\hline $\mathrm{T} / \mathrm{M}$ & . /rar & V/Ifa &.$/ M H$ & 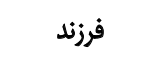 & \\
\hline I/TY & - $/ P M$ & -leqp & $.|+|+1$ & مراقب & \\
\hline- & - & - & - & 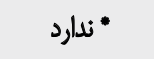 & \\
\hline VYre & MMre & $-1 / M 19$ &.$- / \cdot v$ & دارد & مصرف سيكار \\
\hline Whr & $<+1 .+1$ & 81.90 & . & & حمايت اجتماعي \\
\hline WTO & $\cdot 1 \cdot 1 \mathrm{~A}$ & $-T / R V E$ & $-+/ 1 \pi$ & & ميزان ناتوانىى \\
\hline $1 / 11$ & .1194 & $1 / r \cdot r$ & $\cdot 1 \cdot n$ & & سازكارى هذهبي \\
\hline I/TY & - llar & V/RT &.$/ \cdot 1 p$ & & بهزيستى روانشناختى \\
\hline
\end{tabular}

بودئلد. درمان بيمارىهاى مزمن و بيشكيرى أز آنها كه عامل

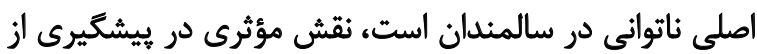

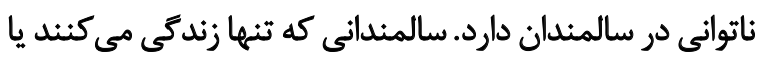

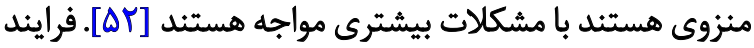

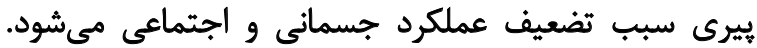

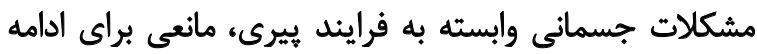

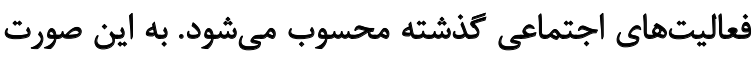

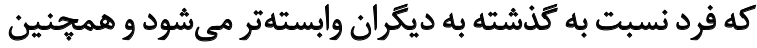

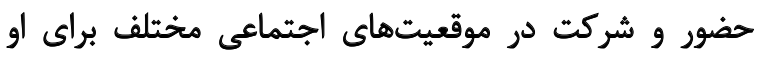

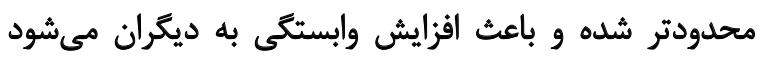

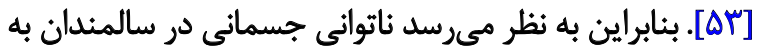

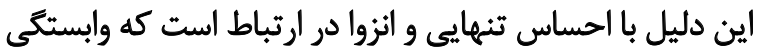

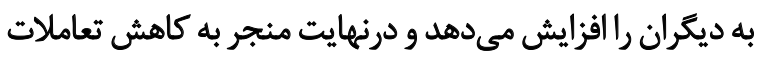

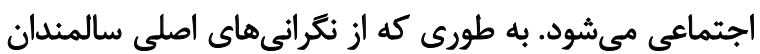
از دست دادن استقلال و وابسته شدن به ديكران است. ترس
احساس بر بهزيستى روانشناختى فرد و درنتيجه بر ميزان

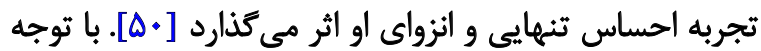

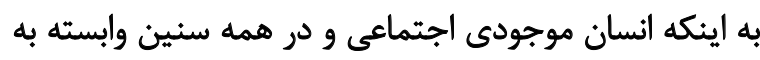

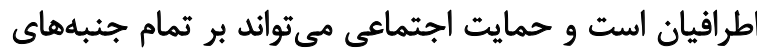

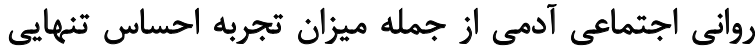

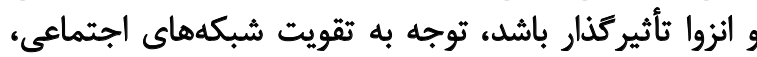

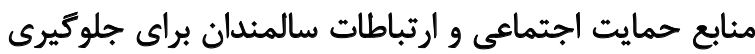

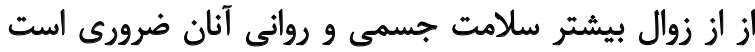

نتايج مطالعه حاضر نشان داد كه ناتوانى از ديكر مثغيرهاي

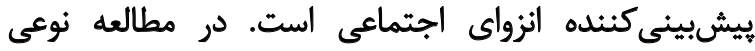

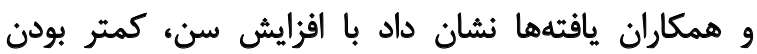

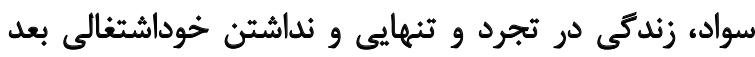

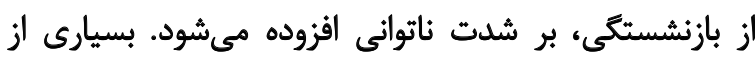

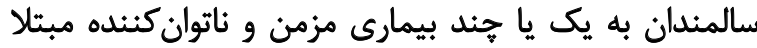


بين بردن يا كاهش اثر ناتوانىهاى جسمانى بر زندكى روزمره

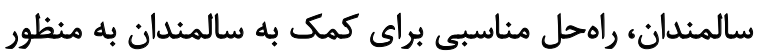

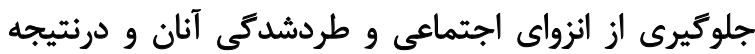
افزايش كيفيت زندئى آنان است.

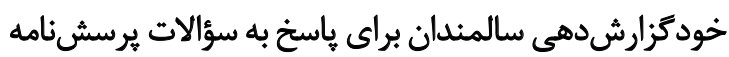

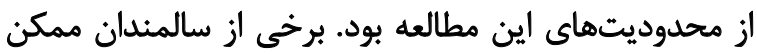

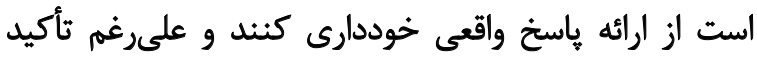

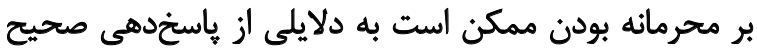

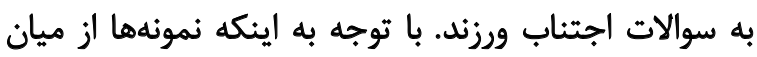

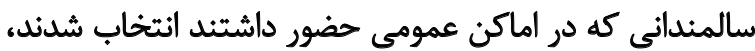

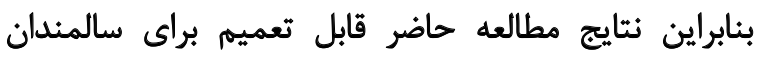

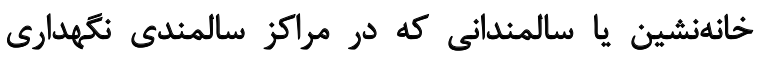

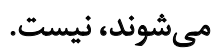

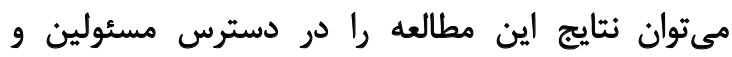

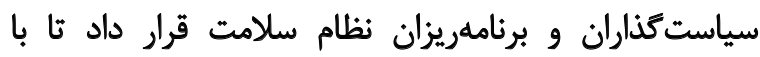

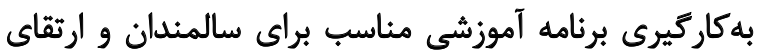

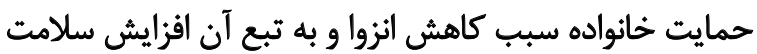
سالمندان و كيفيت زندكى شان شوند انزوان به تبع

بر اساس يافتهها و محدوديتهاى يثروهش حاضر در مورد

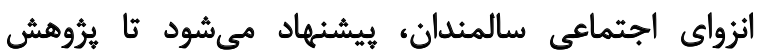

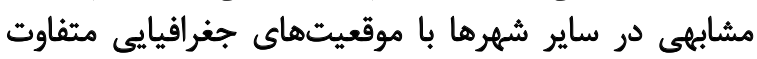

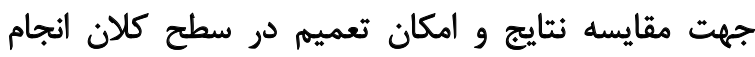

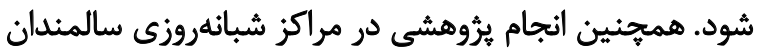

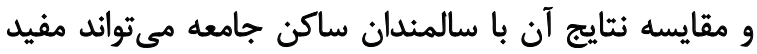

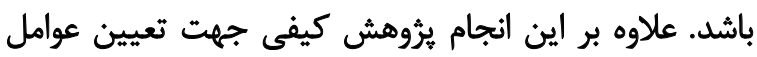

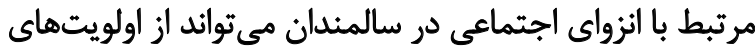

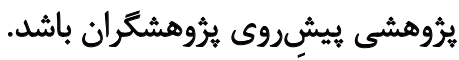

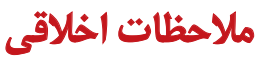

\section{يبيروى ائ اصول اخلاق يثوهش}

ملاحضات اخلاقى در اين مقاله مورد تاييد كميته

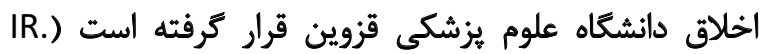

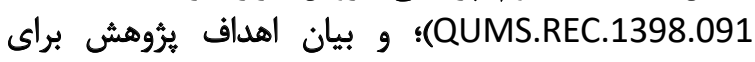

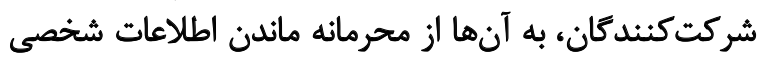

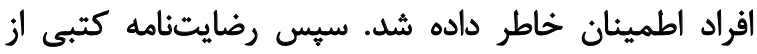

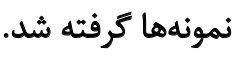

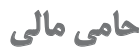

اين يُؤهش بخشى از إياينامه كارشناسى ارشد نويسنده

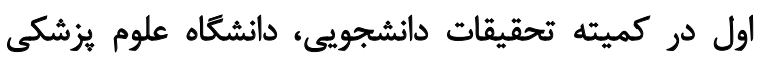

$$
\text { قزوين، قزوين است. }
$$

از سربار بودن سالمند براى خانواده و جامعه باعث به مخاطره

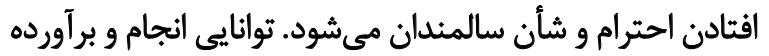

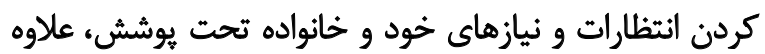

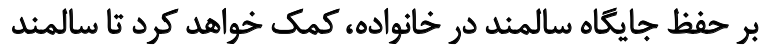

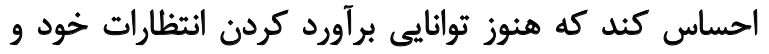

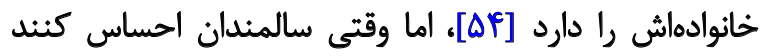

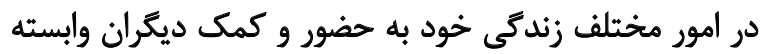

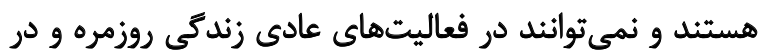

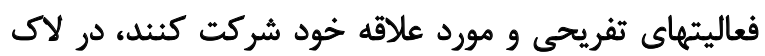

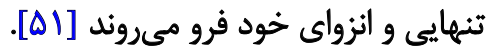
طبق نتايج بهدستآمده انزواى اجتماعى سالمندانى كه

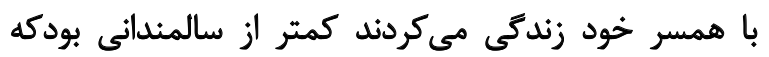

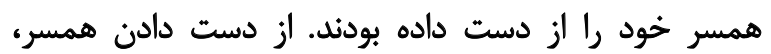

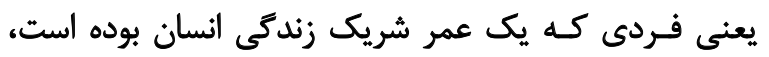

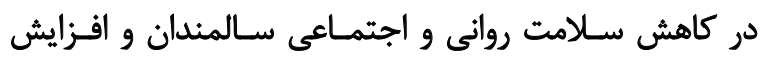

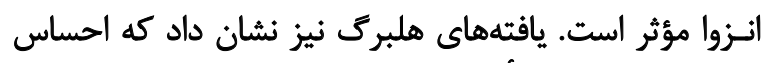

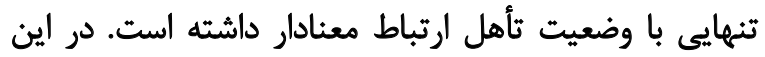

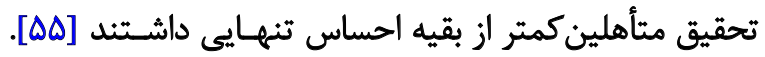

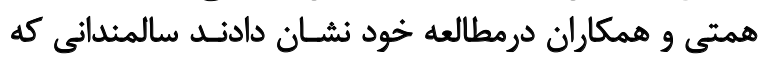

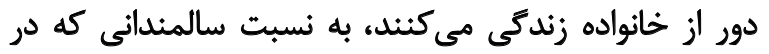

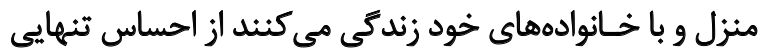

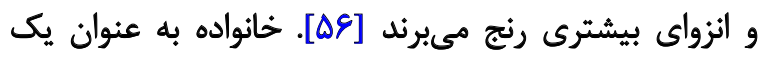

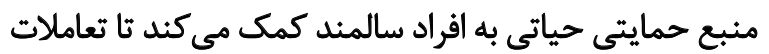

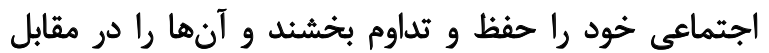

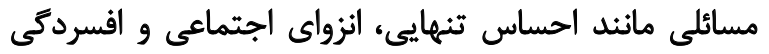

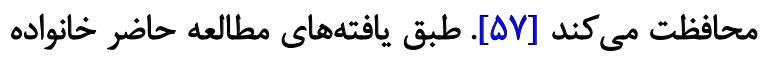

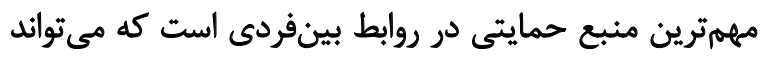
با ارائه حمايت كافى موجب كنيت دنترل و كاهش ائزواي اجتمائي سالمندان باشيد. - ائه كافي

\section{نتيجليَيرى نمهايى}

بر اساس نظريه شبكه، سرمايه اجتماعى باعث به وجود إمود

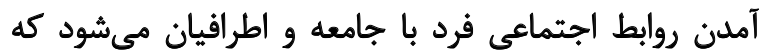

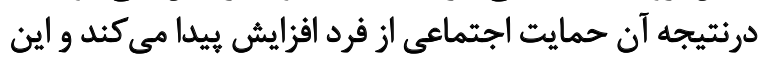

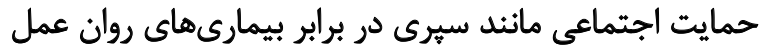

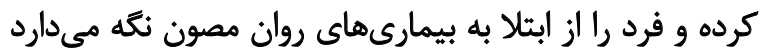

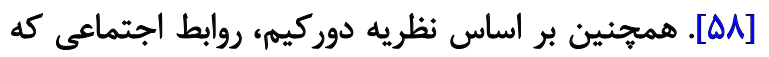

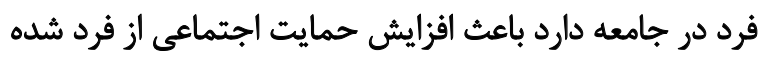

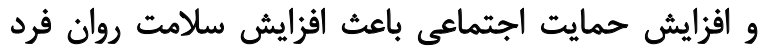

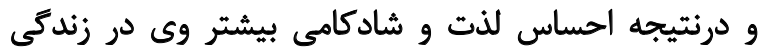

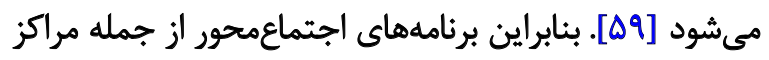

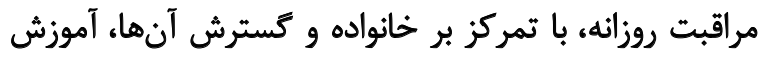

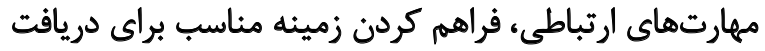

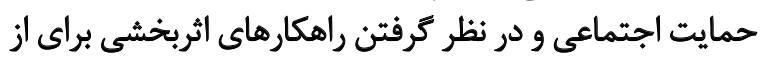




$$
\text { مشار كت نويسندكَان }
$$

مغهومسازى، روششناسى، تحقيق و بروسى، نغارش

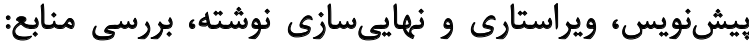

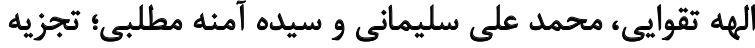

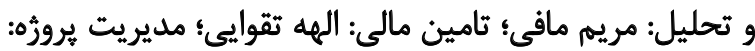

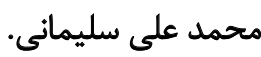

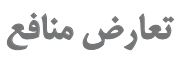

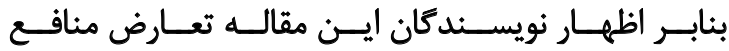
نــدارد.

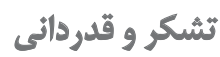

نويسندكان مراتب تشكر و قدردانى خود را از معاونيت

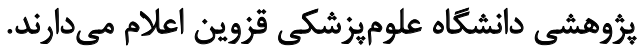




\section{References}

[1] Sadri Damirchi E, Ghomi M, Esmaeli Ghazi Valoii F. [Effectiveness of life review group therapy on psychological well-being and the life expectancy of elderly women (Persian)]. Salmand: Iranian Journal of Ageing. 2017; 12(3):312-25. [DOI:10.21859/ sija.12.3.312]

[2] Alipoor F, Sajadi H, Forozan A, Biglarian A. [The role of social support in elderly quality of life (Persian)]. Social Welfare Quarterly. 2009; 8(33):149-67. http:// refahj.uswr.ac.ir/article1-1862-en.html

[3] Hojjati H, Sharifnia SH, Hosseinalipour S, Akhonzadeh GB, Asayesh $\mathrm{H}$. [The effect of reminiscence on the amount of group self-esteem and life satisfaction of the elderly (Persian)]. Nursing and Midwifery Journal. 2011; 9(5):350-6. http:// unmf. umsu.ac.ir/article-1-607-en.html

[4] Hekmati Pour N, Taheri N, Hojjati H, Rabiee S. [Evaluation of the relationship between social support and quality of life in elderly patients with diabetes (Persian)]. Journal of Diabetes Nursing. 2015; 3(1):42-50. http://jdn.zbmu.ac.ir/article1-106-en.html

[5] Taraghi Z, Ilali ES. [A comparison of older adults' and managers' attitudes towards age-friendly city indexes (Persian)]. Salmand: Iranian Journal of Ageing. 2018; 13(2):236-49. [DOI:10.32598/sija.13.2.236]

[6] Ghazi Mohseni M, Soleimanian AA, Heidarnia A. [Examining the effectiveness of hope-based group training on the life quality of the elderly people (Persian)]. Salmand: Iranian Journal of Ageing. 2016; 11(2):300-9. [DOI:10.21859/sija-1102300]

[7] Bahrami F, Ramezani-Farani A. [Religious orientation (internal and external) effects on aged mental health (Persian)]. Archives of Rehabilitation. 2005; 6(1):42-7. http:/ / rehabilitationj. uswr.ac.ir/article-1-99-fa.html

[8] Heinrich LM, Gullone E. The clinical significance of loneliness: A literature review. Clinical Psychology Review. 2006; 26(6):695-718. [DOI:10.1016/j.cpr.2006.04.002]

[9] Foladian M, Jafari Moghaddam M. [Social policy and the feeling of social isolation in Iranian society (Persian)]. Paper presented at the $4^{\text {th }}$ National Conference on Social and Cultural Research in Iranian society. Tehran, Iran, 2017. https:// profdoc. um.ac.ir/articles/a/1071564.pdf

[10] Zhou G, Wang Y, Yu X. Direct and indirect effects of family functioning on loneliness of elderly Chinese individuals. Current Psychology. 2018; 37(1):295-301. [DOI:10.1007/s12144-0169512-5]

[11] Ejiri M, Kawai H, Fujiwara Y, Ihara K, Hirano H, Kojima M, et al. [Predictors of social isolation among older people living in urban area: A prospective study (Japanese)]. Nihon Kōshū Eisei Zasshi. 2018; 65(3):125-33. [DOI:10.11236/jph.65.3_125] [PMID]

[12] Taylor HO, Taylor RJ, Nguyen AW, Chatters L. Social isolation, depression, and psychological distress among older adults. Journal of Aging and Health. 2018; 30(2):229-46. [DOI:10.1177/0898264316673511]

[13] Cotterell N, Buffel T, Phillipson Ch. Preventing social isolation in older people. Maturitas. 2018; 113:80-4. [DOI:10.1016/j. maturitas.2018.04.014]

[14] Valtorta NK, Kanaan M, Gilbody S, Ronzi S, Hanratty B. Loneliness and social isolation as risk factors for coronary heart disease and stroke: Systematic review and meta-analysis of longitudinal observational studies. Heart. 2016; 102(13):1009-16. [DOI:10.1136/heartjnl-2015-308790]
[15] Matthews GA, Nieh EH, Vander Weele CM, Halbert SA Pradhan RV, Yosafat AS, et al. Dorsal raphe dopamine neurons represent the experience of social isolation. Cell. 2016 164(4):617-31. [DOI:10.1016/j.cell.2015.12.040]

[16] Kuiper JS, Zuidersma M, Oude Voshaar RC, Zuidema SU, van den Heuvel ER, Stolk RP, et al. Social relationships and risk of dementia: A systematic review and meta-analysis of longitudinal cohort studies. Ageing Research Reviews. 2015; 22:39-57. [DOI:10.1016/j.arr.2015.04.006]

[17] Steptoe A, Shankar A, Demakakos P, Wardle J. Social isolation, loneliness, and all-cause mortality in older men and women. Proceedings of the National Academy of Sciences. 2013; 110(15):5797-801. [DOI:10.1073/pnas.1219686110]

[18] Modarresi Yazdi F, Farahmand M, Afshani SAR. [A study on single girls' social isolation and its effective socio-cultural factors: A study on single girls over 30 years in Yazd (Persian)]. Journal of Social Problems of Iran. 2017; 8(1):121-43. http:/ /jspi. khu.ac.ir/article-1-2724-en.html

[19] Ilali ES, Peyrovi H, Sahaf R, Akbari Kamrani AA, Spahbodi F. [Challenges of undergoing dialysis with emphasis on aging syndromes in the elderly (Persian)]. Salmand: Iranian Journal of Ageing. 2017; 11(4):566-71. [DOI:10.21859/sija-1104566]

[20] Victor CR, Scambler SJ, Bowling A, Bond J. The prevalence of, and risk factors for, loneliness in later life: A survey of older people in Great Britain. Ageing \& Society. 2005; 25(6):357-75. [DOI:10.1017/S0144686X04003332]

[21] Savikko N, Routasalo P, Tilvis RS, Strandberg TE, Pitkälä KH. Predictors and subjective causes of loneliness in an aged population. Archives of Gerontology and Geriatrics. 2005; 41(3):223-33. [DOI:10.1016/j.archger.2005.03.002]

[22] Victor Ch, Scambler S, Bond J, Bowling A. Being alone in later life: Loneliness, social isolation and living alone. Reviews in Clinical Gerontology. 2000; 10(4):407-17. [DOI:10.1017/ S0959259800104101]

[23] Umberson D, Karas Montez J. Social relationships and health: A flashpoint for health policy. Journal of Health and Social Behavior. 2010; 51(1_Suppl):S54-66. [DOI:10.1177/0022146510383501]

[24] Visser MA, El Fakiri F. The prevalence and impact of risk factors for ethnic differences in loneliness. European Journal of Public Health. 2016; 26(6):977-83. [DOI:10.1093/eurpub/ ckw115]

[25] Platt L. Social activity, social isolation and ethnicity. The Sociological Review. 2009; 57(4):670-702. [DOI:10.1111/j.1467954X.2009.01867.x]

[26] Lubben J. Addressing social isolation as a potent killer! Public Policy \& Aging Report. 2017; 27(4):136-8. [DOI:10.1093/ppar/ prx026]

[27] Wang YH, Huang FY, Zheng Y, Shi GS, Wang L, Liao SS, [Study on the association between social isolation and cognitive function among elderly in Daqing city, Heilongjiang province (Chinese)]. Zhonghua Liuxingbingxue Zazhi. 2017; 38(4):472-7. [DOI:10.3760/cma.j.issn.0254-6450.2017.04.012] [PMID]

[28] Tomaka J, Thompson Sh, Palacios R. The relation of social isolation, loneliness, and social support to disease outcomes among the elderly. Journal of Aging and Health. 2006; 18(3):359-84. [DOI:10.1177/0898264305280993] [PMID] 
[29] Schutter N, Holwerda TJ, Stek ML, Dekker JJM, Rhebergen D, Comijs HC. Loneliness in older adults is associated with diminished cortisol output. Journal of Psychosomatic Research. 2017; 95:19-25. [DOI:10.1016/j.jpsychores.2017.02.002]

[30] Lubben JE. Assessing social networks among elderly populations. Family \& Community Health. 1988; 11(3):42-52. [DOI:10.1097/00003727-198811000-00008]

[31] Lubben J, Gironda M. Measuring social networks and assessing their benefits. Social networks and social exclusion Sociological and policy perspectives. England, UK: Routledge; 2004. https://www.taylorfrancis.com/chapters/ mono/10.4324/9781315242897-11/measuring-social-networksassessing-benefits-graham-allan-chris-phillipson

[32] Rahmani M, Ghasemi V, Hashemianfar A. [Investigation of the effect of social relations on high-risk behaviors of adolescents of Bojnourd city, Iran (Persian)]. Journal of Applied Sociology. 2016; 27(1):1-26. [DOI:10.22108/JAS.2016.20478]

[33] Zimet GD, Dahlem NW, Zimet SG, Farley GK. The multidimensional scale of perceived social support. Journal of Personality Assessment. 1988; 52(1):30-41. [DOI:10.1207/s15327752jpa5201_2]

[34] Jowkar B, Salimi A. [Psychometric properties of the short form of the Social and Emotional Loneliness Scale for Adults (SELSAS) (Persian)]. International Journal of Behavioral Sciences. 2012; 5(4):311-7. http:/ / www.behavsci.ir/article_67748.html

[35] Carlozzi NE, Kratz AL, Downing NR, Goodnight S, Miner JA Migliore N, et al. Validity of the 12-item World Health Organization Disability Assessment Schedule 2.0 (WHODAS 2.0) in individuals with Huntington Disease (HD). Quality of Life Research. 2015; 24(8):1963-71. [DOI:10.1007/s11136-015-0930-x]

[36] Abedzadeh-Kalahroudi M, Razi E, Sehat M. The relationship between socioeconomic status and trauma outcomes. Journal of Public Health. 2018; 40(4):e431-9. [DOI:10.1093/pubmed/fdy033]

[37] Topp CW, Østergaard SD, Søndergaard S, Bech P. The WHO-5 well-being index: A systematic review of the literature. Psychotherapy and Psychosomatics. 2015; 84(3):167-76. [DOI:10.1159/000376585]

[38] Dehshiri GR, Mousavi SF. [An investigation into psychometric properties of Persian version of World Health Organization five well-being index (Persian)]. Journal of Clinical Psychology. 2016; 8(2):67-75. [DOI:10.22075/JCP.2017.2237]

[39] Pargament K, Feuille M, Burdzy D. The brief RCOPE: Current psychometric status of a short measure of religious coping. Religions. 2011; 2(1):51-76. [DOI:10.3390/rel2010051]

[40] Pargament KI, Koenig HG, Perez LM. The many methods of religious coping: Development and initial validation of the RCOPE. Journal of Clinical Psychology. 2000; 56(4):519-43. [DOI:10.1002/ (SICI)1097-4679(200004)56:4<519::AID-JCLP6>3.0.CO;2-1]

[41] Rajabi M, Saremi AA, Bayazi MH. [The relationship between religious coping patterns, mental health and happiness (Persian)] Developmental Pschology: Iranian Psychologists. 2012; 8(32):36371. http://jip.azad.ac.ir/article_512246.html

[42] Ibrahim R, Abolfathi Momtaz Y, Hamid TA. Social isolation in older Malaysians: Prevalence and risk factors. Psychogeriatrics. 2013; 13(2):71-9. [DOI:10.1111/psyg.12000]

[43] Johnson JE, Waldo M, Johnson RG. Research considerations: Stress and perceived health status in the rural elderly. Journal of Gerontological Nursing. 1993; 19(10):24-9. [DOI:10.3928/00989134-19931001-07]
[44] Yahaya N, AbdullahSS, Momtaz YA, Hamid TA. Quality of life of older Malaysians living alone. Educational Gerontology. 2010 36(10-11):893-906. [DOI:10.1080/03601271003609009]

[45] Seyfzadeh A, Hagighatian M, Mohajerani AA. [The relationship between social isolation and health among the Tehranian elderly (Persian)]. Journal of Education and Community Health. 2017; 4(3):19-25. [DOI:10.21859/jech.4.3.19]

[46] Savikko N. Loneliness of older people and elements of an intervention for its alleviation [MSc. thesis]. Turku: University of Turku; 2008. https:/ / www.utupub.fi/handle/10024/38910

[47] Seyfzadeh A, Haghighatian M, Mohajerani AA. [Economic, social and demographic characteristics and social isolation in senile people (Case study: Tehran city) (Persian)]. Middle Eastern Journal of Disability Studies. 2019; 9:23. http://jdisabilstud.org/ article-1-994-en.html

[48] Kapadia F, Levy M, Griffin-Tomas M, Greene RE, Halkitis SN Urbina A, et al. Perceived social support and mental health states in a clinic-based sample of older HIV positive adults. Journal of HIV/AIDS \& Social Services. 2016; 15(4):417-27. [DOI:10.1080/1 5381501.2016.1212295]

[49] Gong G, Mao J. Health-related quality of life among Chinese patients with rheumatoid arthritis: The predictive roles of fatigue, functional disability, self-efficacy, and social support. Nursing Research. 2016; 65(1):55-67. [DOI:10.1097/NNR.0000000000000137]

[50] Farhadi A, Barazandeh Choqaiee S, Mokhtarpour H, Karam $\mathrm{Kh}$, Daraeezadeh A. [Role of social support, spiritual well-being and self-efficacy in predicting the subjective well-being of the elderly (Persian)]. Aging Psychology. 2015; 1(2):35-48. https://jap. razi.ac.ir/article_335.htm

[51] Mirdrikvand F, Panahi H, Hoseyni Ramaghani NS. [Loneliness of the elderly: The role of communication skills, social support and functional disability (Persian)]. Aging Psychology. 2017; 2(2):103-13. https://jap.razi.ac.ir/article_582.html

[52] Noei H, Sahaf R, Akbari Kamrani AA, Abolfathi Momtaz Y, Pourhadi S, Shati M. [The relationship between gender and disability in the elderly people in Tehran Municipality Pension Organization (Persian)]. Salmand: Iranian Journal of Ageing. 2017; 12(1):6-17. [DOI:10.21859/sija-12016]

[53] Heravi Karimloo M, Anoosheh M, Foroughan M, Sheykhi MT Hajizade E, Seyed Bagher Maddah MS, et al. [Loneliness from the perspectives of elderly people: A phenomenological study (Persian)]. Salmand: Iranian Journal of Ageing. 2008; 2(4):410-20. http:// salmandj.uswr.ac.ir/article-1-53-en.html

[54] Mikaeili N, Rajabi S, Abbasi M. [A comparison of loneliness, mental health and self-efficacy of the elderly (Persian)]. Journal of Social Psychology (New Findings in Psychology). 2012; 7(22):7381. https://www.sid.ir/fa/journal/ViewPaper.aspx?ID=191481

[55] Jakobsson U, Hallberg IR. Loneliness, fear, and quality of life among elderly in Sweden: A gender perspective. Aging Clinical and Experimental Research. 2005; 17(6):494-501. [DOI:10.1007/ BF03327417]

[56] Hemmati Alamdarlou Gh, Dehshiri GR, Shojaie S, Hakimi Rad E. [Health and loneliness status of the elderly living in nursing homes versus those living with their families (Persian)]. Salmand Iranian Journal of Ageing. 2008; 3(2):557-64. http://salmandj. uswr.ac.ir/article-1-90-en.html 
[57] Murphy E. The prognosis of depression in old age. The British Journal of Psychiatry. 1983; 142(2):111-9. [DOI:10.1192/ bjp.142.2.111]

[58] Brettell CB, Hollifield JF. Migration theory: Talking across disciplines. New York, London, Routledge: Taylor \& Francis Group; 2000. https:/ / books.google.com/books?id=7zpB10HrcGMC\&dq

[59] Hemati R, Rahimi Aliabad A. [Studying the relationship between social support and mental health (Case study: Women in Khalkhal city) (Persian)]. Journal of Specialized Social Science. 2014; 11(42):106-19. https://www.sid.ir/fa/journal/ViewPaper. aspx?ID $=253814$ 